\title{
Parietal Lobe Ependymal Tumor
}

National Cancer Institute

\section{Source}

National Cancer Institute. Parietal Lobe Ependymal Tumor. NCI Thesaurus. Code C131575.

An ependymal tumor affecting the parietal lobe of the brain. 